\title{
Postherpetische Neuralgie
}

\section{Bei Zoster gleich mit der Schmerztherapie beginnen}

Gürtelrose nicht ernst zu nehmen, ist ein Fehler. Das gilt auch und besonders für die Schmerztherapie: Sie gehört obligatorisch dazu.

„Das heilt von selber!“ Diese Auffassung zur Gürtelrose (Herpes zoster) ist erstaunlicherweise nach wie vor verbreitet. Doch es gibt jeden Grund, Herpes zoster ernst zu nehmen.

Über zwei bis vier Wochen muss bei einer akuten Zoster-Erkrankung mit signifikanten therapiepflichtigen Schmerzen gerechnet werden. Zuweilen beginnt der Schmerz schon vor den Effloreszenzen. Bestehen solche Schmerzen länger als 90 Tage nach Einsetzen des Hautausschlags, wird von postherpetischer Neuralgie (PHN) gesprochen.

Je älter der Zoster-Patient ist, desto wahrscheinlicher wird eine PHN: Liegt die Rate bei etwa 50 -Jährigen bei $10 \%$, ist bei über 85-jährigen Zoster-Patienten jeder Zweite Opfer der PHN - mit teils brennenden Dauerschmerzen, attackenartigen lanzinierenden Schmerzen und/ oder Allodynie/Hyperpathie. Hinzu kommt ein zum Teil quälender Juckreiz.

\section{Innerhalb von 72 Stunden reagieren}

All dies sind Gründe, möglichst unmittelbar nach Auftreten der typischen Bläschen mit der antiviralen Behandlung zu beginnen, spätestens innerhalb von 72 Stunden. In erster Linie werden dazu Aciclovir oder Brivudin genutzt, bei schweren Fällen wird die intravenöse Gabe nötig.

Die topische Behandlung beschleunigt das Eintrocknen der Bläschen, mindert den Juckreiz und beugt der sekundären bakteriellen Infektion vor. Diese erfordert unter Umständen die gezielte Antibiose.

„Was allzu häufig versäumt wird, ist eine ausreichende Schmerztherapie", sagt Dr. Oliver Emrich, Allgemeinarzt am Schmerzzentrum Ludwigshafen. „Sie sollte frühzeitig eingeleitet werden", empfiehlt der Vizepräsident der Deutschen Gesellschaft für Schmerzmedizin (DGS).

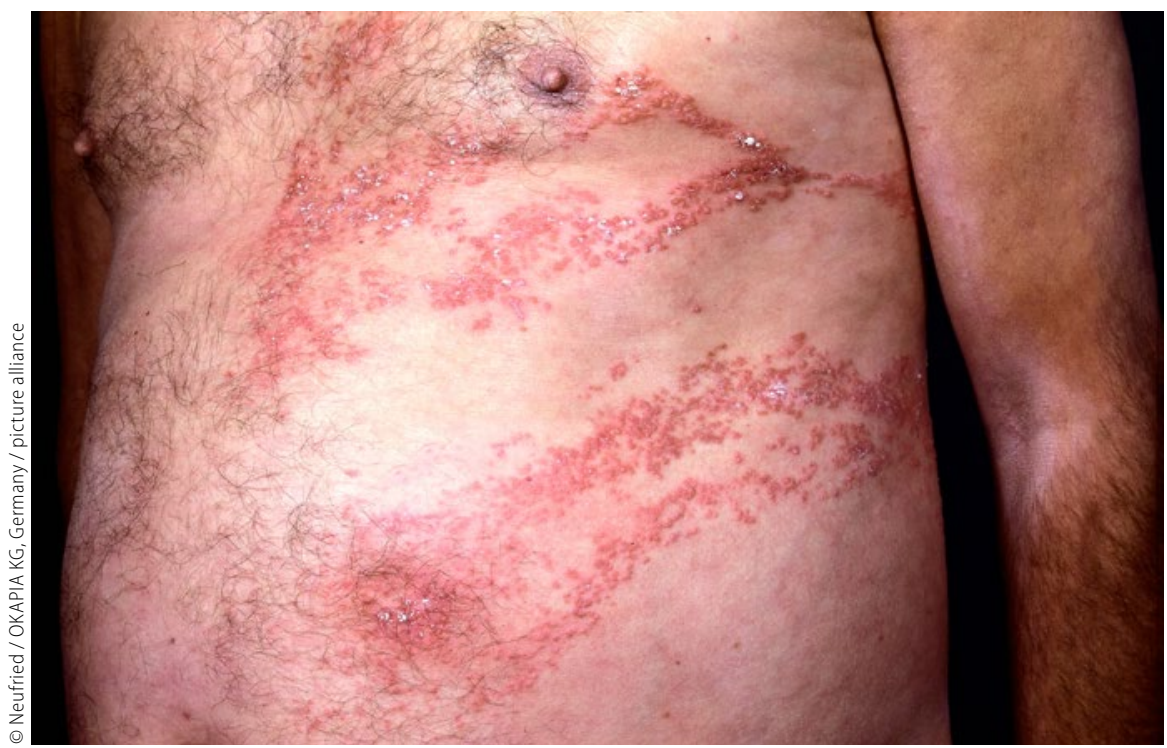

Patient mit Herpes Zoster im Brust- und Bauchbereich.

Ältere Empfehlungen, dabei nach WHOSchema vorzugehen, sind überholt. „Der Schmerz wird von vornherein behandelt wie ein neuropathischer Schmerz", betont Emrich. „Ich beginne mit einem trizyklischen Antidepressivum, etwa Amitryptilin."

Lidocain- oder Polidocanol-haltige Salben und Auflagen haben eine lokal anästhesierende Wirkung. Bei sehr starkem Erstschmerz, vor allem bei Patienten über 60, beginnt Emrich dagegen sofort mit einer niedrig dosierten Dreierkombination aus Amitryptilin, Gabapentin oder Pregabalin und einem Opioid wie Tramadol oder Tilidin. „Damit bestehen die besten Chancen, einer Schmerzchronifizierung vorzubeugen."

Die Schmerztherapie erfolgt nach Leitlinien der Deutschen Gesellschaft für Neurologie. Mittel der ersten Wahl sind trizyklische Antidepressiva, gegebenenfalls selektive Serotonin-(Noradrenalin-) Wiederaufnahmehemmer (SSRI/SNRI) wie Duloxetin sowie die Kalziumkanalmodulierenden Antikonvulsiva Gabapentin oder Pregabalin und/oder - vor allem bei einschießenden Schmerzen Carbamazepin als Natriumkanal-modulierendes Antikonvulsivum.

\section{Individuelle Dosis entscheidend}

„Wichtig ist, die individuelle Dosis mit zu Beginn niedrigen Medikamentenkonzentrationen $\mathrm{zu}$ titrieren, Ziel ist eine mindestens 30 - bis 50\%ige Schmerzlinderung“, sagt Emrich. „Dieses Setting kann von nichtmedikamentösen und lokalen Verfahren flankiert werden." Dazu gehören topische Verfahren mit Lidocain oder ein Versuch mit Capsaicin-Pflastern sowie Lokal- und Leitungsanästhesien. Eine transkutane elektrische Nervenstimulation (TENS) muss supraläsionell oder kontralateral erfolgen, keinesfalls im Schmerzgebiet. 